\title{
Combination of platelet rich fibrin and carbonate hydroxyapatite alloplastic bone graft as periodontal tissue engineering in management of chronic periodontitis: a case report
}

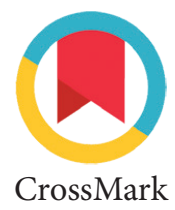

\author{
Arni I. Djais, Nurfaisah
}

\section{Abstract}

Objective: Chronic periodontitis lesions include loss of attachment and bone and are regarded as irreversible. Conventional procedures cannot restore the periodontal tissue to its original form. Periodontal tissue engineering is regenerativemedicine novel therapy by either implanting biomaterials. Platelet Rich Fibrin (PRF) as second generation platelet and Carbonate Hydroxyapatite (CHA) alloplastic bone graft can be used in surgical therapy because ease, inexpensive method and does not need any addition of exogenous compounds and a second surgical site and procedure. This case report aims to evaluate the clinical and radiographic effectiveness combination of both for the treatment of chronic periodontitis.
Methods: A 21-year-old female was reported with chief complaint tooth mobility. Initial examination revealed grade two mobility of 35 with $9 \mathrm{~mm}$ distobuccal and distolingual pocket depth. The Periapical radiograph revealed a large diffused periapical radiolucency in relation to 35. Periodontal surgery was done and the osseous defect was filled with CHA bone graft and PRF.

Results: There was a significant change in pocket depth and grade of mobility six months after surgery to $3 \mathrm{~mm}$ and grade one mobility. Radiographs showed resolution of an osseous defect.

Conclusion: It was concluded that combined PRF with CHA bone graft can be used to treat chronic periodontitis as simple technique periodontal tissue engineering.
Department of Periodontic, Faculty of Dentistry, Hasanuddin University, Makassar, Indonesia
*Correspondence to: Arni I. Djais, Department of Periodontic, Faculty of Dentistry, Hasanuddin University, Makassar, Indonesia nurfaisah@ymail.com

Received: 20 June 2018 Revised: 20 June 2018 Accepted: 17 July 2018 Available Online: 1 April 2020

Keywords: Chronic Periodontitis, Tissue engineering, PRF, CHA, Bone Graft

Cite this Article: Djais Al, Nurfaisah. 2020. Combination of platelet rich fibrin and carbonate hydroxyapatite alloplastic bone graft as periodontal tissue engineering in management of chronic periodontitis: a case report. Journal of Dentomaxillofacial Science 5(1): 62-65. D0I: 10.15562/ jdmfs.v5i1.753

\section{Introduction}

Periodontal disease is a chronic inflammatory condition of the periodontium that is characterized by irreversible destruction. ${ }^{1}$ Conventional treatments,guided tissue regeneration (GTR) and growth factorsfrequently cannot restore the periodontal tissue to its original form and usually cause aesthetic and functional problems. ${ }^{2,4}$

Tissue-engineering (TE) approaches have been recognized to combine a scaffold with living cells or biologically active molecules to form a "tissue-engineering construct", in the presence of adequate blood supply, promotes the repair or regeneration of tissues ${ }^{3}$ TE described as a therapy replacing or regenerating human cells, tissues, or organs to restore or establish their normal function. The treatment components are often used in combinations; replace the missing portion of alveolar bone ("bone grafts"); cover the alveolar bone loss area, protecting it from epithelial down growth ("barriers"); and materials with biological activity that can be administered directly to the defect ("biologics" or "cell therapy"). ${ }^{4}$ Recent developments in the science, technology, engineering, and mathematics (STEM) arena allow for alternatives to address several challenges in the treatment of periodontal disease. ${ }^{5}$

Platelet Rich Factor (PRF) membrane release of key growth factors for at least one week and up to 28 days, an anti-infectious action and aid in immune regulation, promote angiogenesis and serve as a biological healing matrix by supporting cell migration and cytokine release. ${ }^{6}$ PRF has been used in dentistry in various clinical setting. ${ }^{7}$ Much investigation now supports the use of PRF for periodontal and soft tissue repair. ${ }^{8}$ Clinically significant improvements in periodontal parameters when infra-bony defects were treated with PRF alone when compared to open flap debridement. ${ }^{9}$ In addition to standard grafting procedures using bone-derived materials, a number of natural and syntheticmaterials have become commercially available for use in orasurgery. ${ }^{10}$

This case reported the used of periodontal bone grafting materials with carbonate hydroxyapatite (CHA) alloplastic bone graft as the scaffold in combination with PRF as bioactive molecules for a simple and low-cost method TE therapy. 


\section{Case Report}

A 21-year-old female was reported to Department of Periodontics Faculty of Dentistry Hasanuddin University, Makassar, Indonesia with chief complaint tooth mobility and calculus. Patient has a habit of chewing only on the right side. Initial examination revealed grade two mobility of 35 with $9 \mathrm{~mm}$ distobuccal pocket depth figure 1 and $9 \mathrm{~mm}$ distolingual pocket depth. The Periapical radiograph revealed a large diffused periapical radiolucency reaching one-third of the tooth root 35. Scaling and root planing was performed and surgery was done a week later.
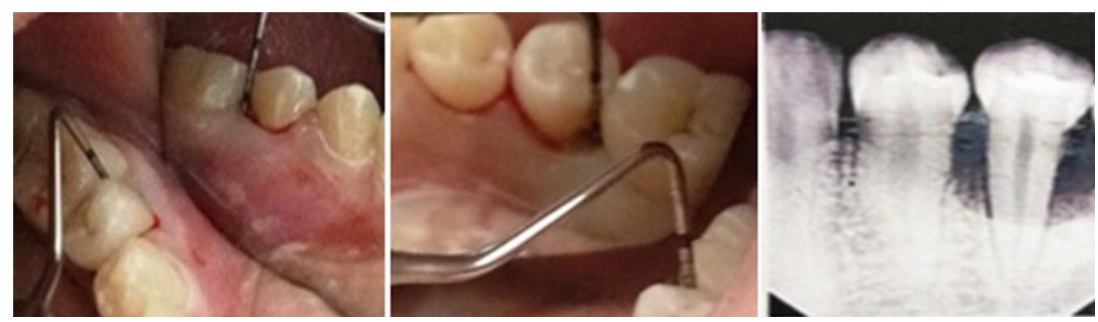

Figure 1 Initial examination
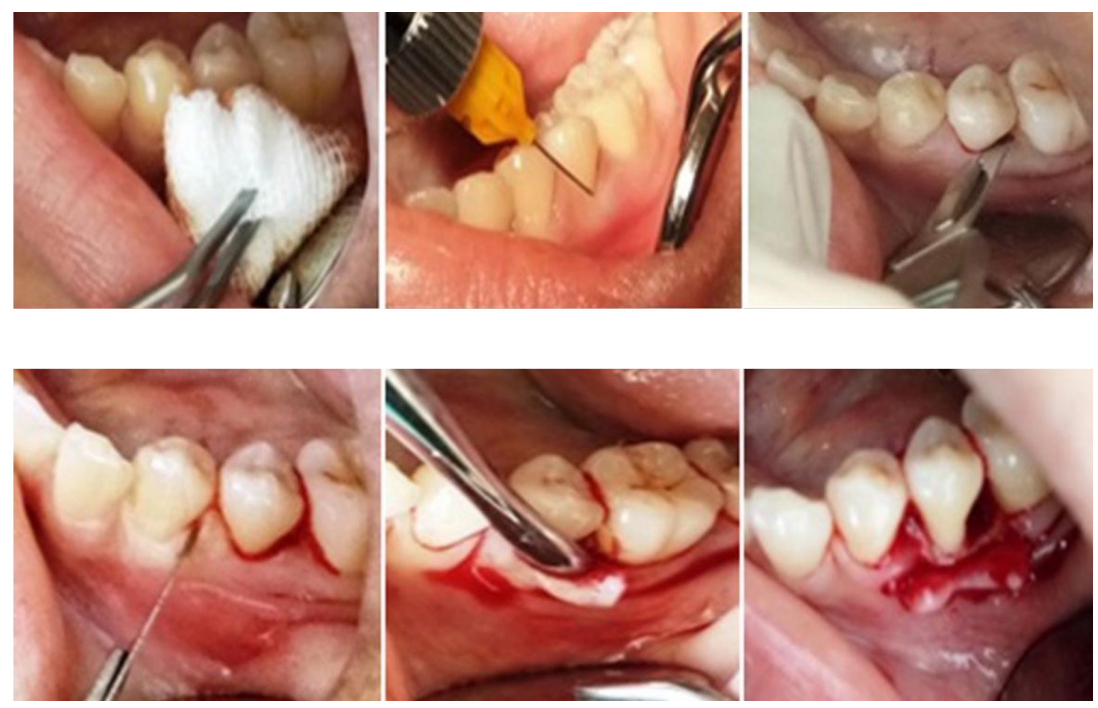

Figure 2 Surgical procedure

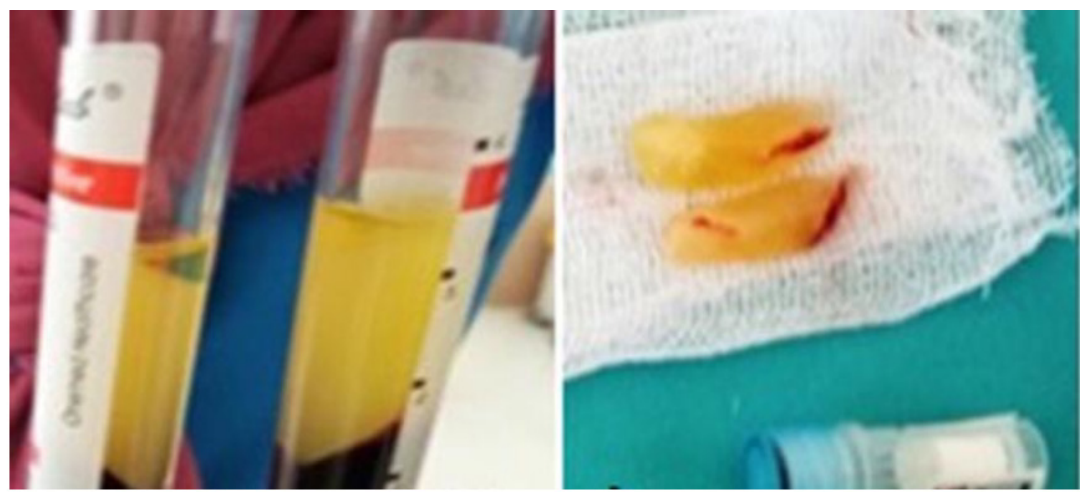

Figure 3 PRF \& Bone graft
The intraoral antisepsis was carried out using iodine solution. A full thickness mucoperiosteal flap was reflected under local anesthesia by a sulcular then vertical incision figure 2. Thorough tissue curettage was done at the defect site.

\section{Preparation of PRF}

The protocol for PRF preparation includes collection of $5 \mathrm{ml}$ whole venous blood in each of the two sterile tubes without anticoagulant then placed in a centrifugal machine at 3,000 rpm for $10 \mathrm{~min}$. There is three layers: Upper straw-colored acellular plasma (platelet-poor plasma), red-colored lower fraction containing red blood cells, and the middle fraction containing the fibrin clot figure 3 . The middle fraction is collected, $2 \mathrm{~mm}$ below to the lower dividing line, which is the PRF. ${ }^{8}$ PRF membrane should be used immediately after preparation as it will shrink, resulting in dehydration and altering the structural integrity of PRF. Dehydration also results in the decreased growth factor content in PRF and leukocyte viability will be adversely affected, altering its biologic properties. ${ }^{9}$

Bone defect was filled with Bone graft figure 3 then applied PRF above it The mucoperiosteal flaps were repositioned and secured in place by giving simple interrupted suture and a periodontal dressing was given figure 4 .

Postoperative instructions, amoxicillin $500 \mathrm{mg}$, ibuprofen $400 \mathrm{mg}$ and $0.2 \%$ chlorhexidine gluconate rinses were prescribed. One week postoperatively, no gingival edema, erythema, suppuration, patient discomfort, or flap dehiscence.

Follow up six months after surgery showed significant improvement of clinical outcomes where the depth of the distobuccal and distolingual pocket was reduced to from $9 \mathrm{~mm}$ to $3 \mathrm{~mm}$ and grade mobility decreased from grade two mobility to grade one mobility.

Periapical radiography showed significant bone formation characterized by a decrease in radiolucency areas reaching a half of the root of the tooth 35 figure 5 .

\section{Discussion}

In general, tissue engineering combines three key elements, namely scaffolds (collagen, bone mineral), signaling molecules (growth factors), and cells (osteoblasts, fibroblasts). ${ }^{8}$ Natural or artificial scaffolds play an important role in growth factor storage and space maintenance for tissue reconstruction and in stem cell proliferation and differentiation. ${ }^{10}$

PRF is considered a second-generation autologous platelet concentrates inducing the proliferation, differentiationand migration of cells responsible 

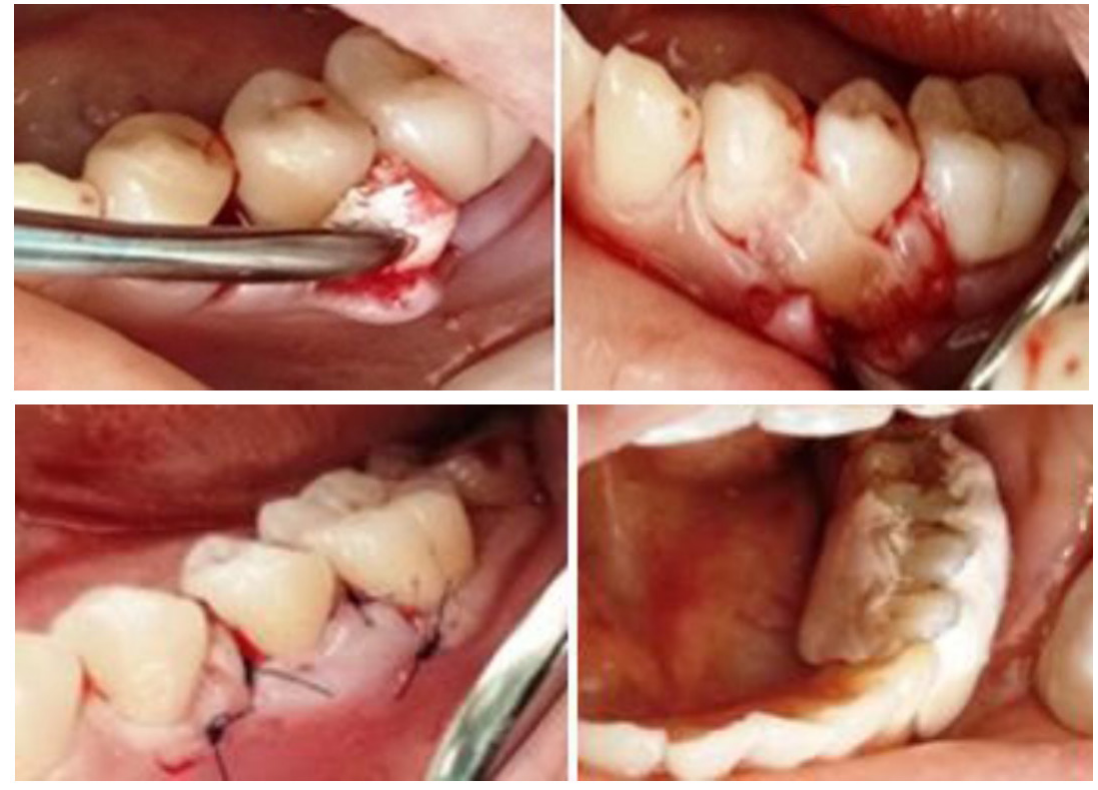

Figure 4 Application bone graft, PRF, Suturing and dressing
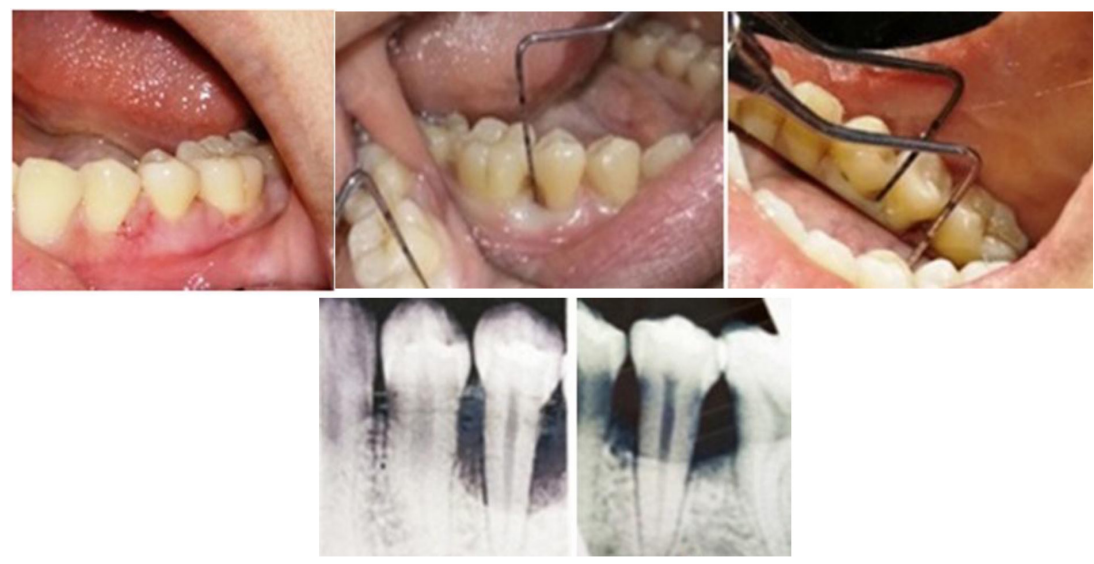

Figure 5 A week post surgery, Six months after surgery, Periapical radiography before and six months after surgery

for periodontal regeneration. ${ }^{11}$ PRFconsists of a fibrin matrix polymerized with the incorporation of platelets, leukocyte, and cytokines, and circulating stem cells with a specific slow release of growth factors and glycoproteins. ${ }^{12}$ An intermediate layer after centrifuge called "buffy coat" or L-PRF where most leucocytes and platelets are concentratedthat stimulates the local environment for differentiation and proliferation of stem and progenitor cells. It acts as an immune regulation node with inflammation control abilities. ${ }^{13}$

PRF membranes protects the surgical site; promotes soft tissue healing; and when its fragments mixes with graft material, it functions as a "biological connector" between the different elements of graft and acts as a matrix which supports neoangiogenesis, capture of stem cells, and migration of osteoprogenitor cells to the center of graft. ${ }^{11}$
Alloplastic do not provide any osteogenic cells or osteoinductive proteins, they are considered osteoconductive only. ${ }^{14} \mathrm{CHA}$ has been proposed as an alternative material for bone regeneration not only due to its similarity to the mineral phase of calcified tissue, but mainly because this material may overcome limitations related to crystallinity and low solubility, and therefore extend the therapeutic capacity of HA.CHA more bioactive and soluble than Hydroxyapatite,contains nanostructured as well as a naturalized collagenized polymer which serves to accelerate the regeneration process of bone tissue, possesses osteoconduction, osteoinduction, and osteogenesis properties. CHA is a major component of human bones produced synthetically but identically (similarly and simultaneously) so as to avoid the immunological reactions. ${ }^{18,15}$

Guided bone regeneration (GBR) principle inspired from GTR principle postulates that specific external additional cells are required to augment inhabiting cells so that new tissue regeneration can be accelerated. Osteoblast cells with ability to promote new alveolar bone remodeling are also required for GBR. ${ }^{20}$

\section{Conclusion}

TE make cost-effective, patient-specific treatment options that provide maximal function and esthetics.PRF can be used in conjunction with CHA alloplastic bone grafts, which offers several advantages including promoting wound healing, bone growth and maturation, graft stabilization, wound healing and hemostasis, and improving the handling properties of graft materials.

\section{Acknowledgment}

The authors would like to acknowledge the support from nurses, laboratory analysts and resident department of periodontic dental and mouth hospital Hasanuddin University, Makassar, Indonesia.

\section{Conflict of Interest}

The authors report no conflict of interest.

\section{References}

1. Han J, Menicanin D, Gronthos S, et al. Stem cells, tissue engineering and periodontal regeneration. Aust Dent J. 2014;17: 117-130.

2. Iwata T, Yamato M, Ishikawa I, et al. Tissue engineering in periodontal tissue. The anatomical Record 2014;25: 16-25.

3. Babo PS, Reis RL, Gomes ME. Periodontal tissue engineering: current strategies and the role of platelet rich hemoderivatives. J Mater Chem B 2017;5: 3617-3628. 
4. Ivanovski S, Vaquette C, Gronthos S, et al. Multiphasic scaffolds for periodontal tissue engineering. J Dent Res 2014;XX: 1-10.

5. Giannobile W V. Regenerative medicine for periodontal and peri-implant diseases.J Dent Res 2015: 1-12.

6. Carter SSD, Costa PF, Vaquette C, et al. Additive biomanufacturing: an advanced approach for periodontal tissue regeneration. Ann Biomed Eng 2017;45: 12-22.

7. Kumar G, Mds P, Kumar S, et al. Platelet Rich Fibrin (PRF) in regeneration of intrabony defects-a randomized controlled trial. J Periodontol 2017: 1-14.

8. Miron RJ, Zucchelli G, Pikos MA, et al. Use of platelet-rich fibrin in regenerative dentistry: a systematic review. Clin Oral Investig 2017;21: 1913-1927.

9. Shah M, Deshpande N, Bharwani A, et al. Effectiveness of autologous platelet-rich fibrin in the treatment of intra bony defects: A systematic review and meta-analysis. J indian Soc periodontol2014;18: 698-704.

10. Najeeb S, Khurshid Z, Agwan MAS, et al. Regenerative potential of platelet rich fibrin (prf) for curing intrabony periodontal defects: a systematic review of clinical studies. Tissue Eng Regen Med. 2017;14: 735-742.

11. Verma A, Srivastava S, Khurshid S, Parveen F, Pandey P. Platelet Rich Fibrin : a promising innovation in regenerative. J Evolution of Med and Dent Sci. 2015;4: 5748-5756.

12. Agrawal I, Chandran S, Nadig P. Comparative evaluation of the efficacy of platelet rich fibrin and calcium phosphosilicate putty alone and in combination in the treatment of intrabony defects : a randomized clinical and radiographic study. Contemp Clin Dent 2018;8: 205-210.

13. Ogawa K, Miyaji H, Kato A, et al. Periodontal tissue engineering by nano beta- tricalcium phosphate scaffold and fibroblast growth factor-2 in one-wall infrabony defects of dogs. J Periodontal Res 2016: 1-10.
14. M DF, Panda S, Nd J, et al. Autologous platelet concentrates for treatment of periodontal defects (Protocol).Cochrane Database Syst Rev 2014;12: 1-8.

15. Gk P, Pc D, Sisodia N, et al. Platelet rich fibrin in management of complex endoperio cases. Kathmandu Univ Med J 2017;15: 101-104.

16. Castro AB, Meschi N, Temmerman A, et al. Regenerative potential Leucocyte-platelet rich fibrin (L-PRF) part A intrabony defects, furcation defects, periodontal Plast surgery A Syst Rev meta-analysis. 2016.

17. Bartold PM, Gronthos S, Ivanovski S, Fisher A, Hutmacher DW. Tissue engineered periodontal products. J Periodontal Res. 2016;51: 1-15.

18. Calasans-maia MD, Raposo B, Guldqd DM. Cytocompatibility and biocompatibility of nanostructured carbonated hydroxyapatite spheres for bone repair. J Appl Oral Sci 2015;23: 599-608.

19. Surbakti A, Oley MC, Prasetyo E. The comparison between carbonate apatite and hydroxy apatite use in the process of closing the calvarian defect using platelet rich plasma.J Biomedic 2017;9: 107-114.

20. Anton, Rahajoe PS, Dwirahardjo B. Sandwich Bone Augmentation (SBA) in immediate implant placement post-dentoalveolar trauma: a case report. J Dentomaxillofac Sci 2017;2: 197-200.

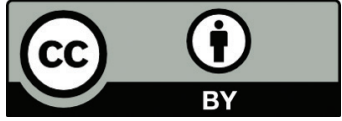

This work is licensed under a Creative Commons Attribution 